Research Article

\title{
Computational Study on the Biomechanics of Pupil Block Phenomenon
}

\author{
Wenjia Wang $\mathbb{D D}^{1,2,3}$ Hongfang Song $\mathbb{D}^{1,2}$ and Zhicheng Liu $\mathbb{D}^{1,2}$ \\ ${ }^{1}$ School of Biomedical Engineering, Capital Medical University, Beijing, China \\ ${ }^{2}$ Beijing Key Laboratory of Fundamental Research on Biomechanics in Clinical Application, Beijing, China \\ ${ }^{3}$ Beijing Tiantan Hospital, Capital Medical University, Beijing, China \\ Correspondence should be addressed to Hongfang Song; songhf@ccmu.edu.cn and Zhicheng Liu; zcliu@ccmu.edu.cn
}

Received 25 March 2019; Revised 28 July 2019; Accepted 23 August 2019; Published 25 September 2019

Academic Editor: Hwa-Liang Leo

Copyright (C) 2019 Wenjia Wang et al. This is an open access article distributed under the Creative Commons Attribution License, which permits unrestricted use, distribution, and reproduction in any medium, provided the original work is properly cited.

Pupil blocking force (PBF) can indicate the potential risk of pupil block (PB), which is considered as a main pathogenic factor of primary angle-closure glaucoma (PACG). However, the effect of PB on the PBF under different pupil diameters and iris-lens channel (ILC) distance was unknown. Besides, a simple and practical method to assess PBF has not been reported yet. In this study, 21 finite element models of eyes with various pupil diameters $(2.4 \mathrm{~mm}-2.6 \mathrm{~mm})$ and ILC $(2 \mu \mathrm{m}-20 \mu \mathrm{m})$ were constructed and were conducted to simulate aqueous humor flow by fluid-solid coupling numerical simulation. PBF in each model was calculated based on the numerical simulation results and was fitted using response surface methodology. The results demonstrated that ILC distance had a more significant effect than pupil diameter on PBF. With the decrease of ILC distance, the PBF increased exponentially. When the reduced distance was lower than $5 \mu \mathrm{m}$, the PBF exploded quickly, resulting in a high risk of iris bomb. The PBF also varied with pupil diameter, especially under the condition of narrow ILC. Both ILC distance and pupil diameter could explain more than $97 \%$ variation in $\mathrm{PBF}$, and a second-order empirical model has been developed to be a good predictor of PBF. Based on the linear relationship between anterior chamber deformation and PBF, a threshold value of PBF was given to guide clinical decisions. This study could be used to investigate PACG pathological correlation and its pathogenesis, so as to provide a reference value for clinical diagnosis of PACG.

\section{Introduction}

Glaucoma is the leading cause of incurable blindness worldwide [1-5]. The most common clinical type in East Asia is primary angle-closure glaucoma (PACG) [6-8], which is characterized by a narrow anterior chamber angle (ACA) [9]. $\mathrm{ACA}$ is considered as the outlet of aqueous humor $(\mathrm{AH})$ in the eye. Once the narrowing of ACA occurred, the outflow of $\mathrm{AH}$ would be blocked, resulting in an obvious increase in intraocular pressure (IOP), which could cause progressive damage to the structure and function of the optic nerve [10]. Therefore, the maintenance of IOP is critical to the stability of eyeball structure and eye health $[11,12]$.

IOP is regulated by the flow of aqueous humor $(\mathrm{AH})$ [13]. AH is secreted by the ciliary body continuously, flows from the posterior chamber (PC) into the anterior chamber
(AC) through the pupil, and drains through the trabecular meshwork (Figure 1). Under the normal physiological condition, the rate of $\mathrm{AH}$ production is equal to that of drainage, maintaining a stable IOP in the eye. However, many factors can block $\mathrm{AH}$ flowing and the most common one is the pupillary block (PB) in PACG patients [14]. Under $\mathrm{PB}$ physiological condition, iris-lens channel (ILC) became narrow, which increased the resistance in the flow pathway from $\mathrm{PC}$ to $\mathrm{AC}$ and hence resulted in a higher IOP in PC and consequently increased the pressure difference between PC and AC [15]. Finally, the elevation of the differential pressure $\left(P_{\mathrm{d}}\right)$ would push iris anteriorly and further narrow ACA, which eventually caused a high IOP and further an irreversible damage to the optic nerve.

To quantify the effect of $\mathrm{PB}$ on the resistance of $\mathrm{AH}$ flow, pupil blocking force $(\mathrm{PBF})$ has been proposed to represent 


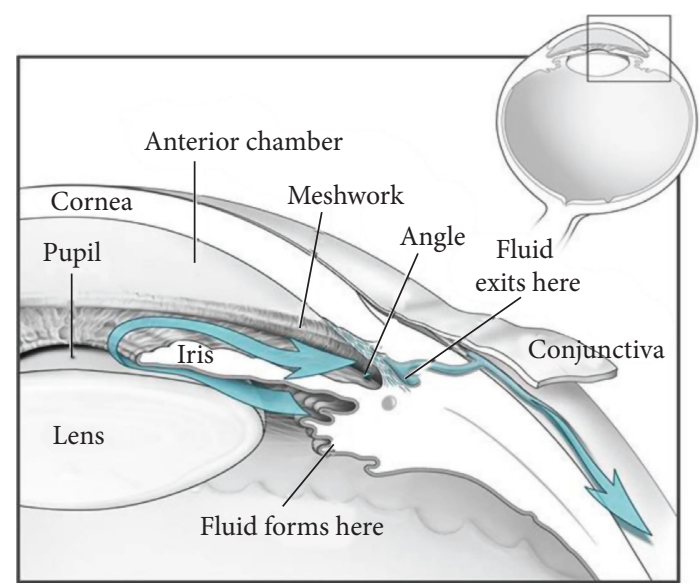

Figure 1: Diagram of the anterior segment of the eye (courtesy: National Eye Institute, National Institutes of Health, Bethesda, $\mathrm{MD})$.

that $\mathrm{AH}$ encountered finite resistance in passing through the narrow ILC. By assuming that iris was a type of linear elastic material, a formula based on Hooke's law to calculate PBF has been deduced in early research $[16,17]$. However, this method only included the limited physiological influencing factors of PBF and neglected the main resistance in ILC, i.e., $\mathrm{AH}$ flow resistance. A method based on computational fluid dynamics (CFD) has been successfully used to study $\mathrm{AH}$ flow under PB condition and visualize the flow field [18]. CFD is a powerful tool to analyze the biofluid flow as well as mechanical distribution, and it has been widely used in biomechanics. Therefore, PBF could be assessed precisely by using CFD technology.

As a biomechanical phenomenon, PBF could be affected by a variety of factors. Wang et al. compared the PB degree under different lens' relative position and revealed that lens' position could affect PBF significantly [19]. Huang and Barocas found that lens curvature also had an obvious effect on PBF by setting an active sphincter to simulate PB conditions [9]. Besides, other factors such as AH flow through the iris-lens passage [20,21] and lens size [22] were proved to be the effect factors of PBF. However, only one factor was investigated in most of the previous research, and a systematic study of various factors has not been reported yet. Moreover, despite high importance, a credible calculation method of PBF was still unknown. Therefore, it was necessary to investigate the comprehensive effect of several factors including pupil diameter, eye size, AC depth, lens size, and its location on PBF as well as AH flow and further construct a computational method to evaluate PBF.

The aim of this study was to investigate the effect of different optical conditions on PBF and construct a model to evaluate PBF. Firstly, twenty-one eye models with different pupil diameters and ILC were constructed and were conducted with numerical simulations. Then, PBF was calculated based on a modified Bernoulli's equation. After that, response surface methodology was utilized to fit the relationship between PBF and pupil diameter as well as ILC distance. The effect of pupil diameter, ILC distance, and their interaction on PBF was also discussed. Finally, the AC morphology including variation in ACA and iris deformation under different $\mathrm{PBF}$ was investigated and a threshold value of PBF was given to guide clinical decisions.

\section{Methods}

2.1. Pupil Blocking Force. AH mainly flows from PC to AC through ILC. Assuming that AH was ideal fluid, its mechanical energy should remain constant at any position based on Bernoulli's equation. However, due to complex components, the viscidity of AH could not be neglected [23]. Considering the fluid viscosity, Bernoulli's equation could be modified as

$$
p+\frac{1}{2} \rho v^{2}+\rho g h+h_{\mathrm{f}}=C,
$$

where $p$ is the pressure at one position, $h$ is its height, $\rho$ is the fluid density, $v$ is the fluid velocity, $g$ is the acceleration of gravity, $h_{\mathrm{f}}$ represents the flow resistance during $\mathrm{AH}$ flow pathway, and $C$ is constant. When AH flows through ILC (Figure 2), the following equation could be induced to describe the conservation of energy:

$$
0+\frac{1}{2} u_{1}^{2}+\frac{p_{1}}{\rho}=Z g+\frac{1}{2} u_{2}^{2}+\frac{p_{2}}{\rho}+H_{\mathrm{f}}
$$

where $u_{1}$ and $u_{2}$ are the average velocities in the two surfaces $\left(S_{1}\right.$ and $\left.S_{2}\right), p_{1}$ and $p_{2}$ are the pressures in these surfaces, $A_{1}$ and $A_{2}$ are their areas, respectively, $Z$ is the vertical distance from $S_{1}$ to $S_{2}$, and $H_{\mathrm{f}}$ (unit of $\mathrm{J} / \mathrm{kg}$ ) is the resistance (i.e., $\mathrm{PBF}$ ) when AH flows from $S_{1}$ to $S_{2}[14,24]$.

Known that $\mathrm{AH}$ is secreted by the ciliary body, its volumetric flux (represented by $Q$ ) in the surfaces $S_{1}$ and $S_{2}$ is identical and can be calculated as the multiplication by area and velocity; therefore, the following equation can be obtained:

$$
Q=S_{1} \cdot u_{1}=S_{2} \cdot u_{2}
$$

By applying equation (3), equation (2) can be written as equation (4) and PBF can be calculated based on the results of pressure and area:

$$
H_{\mathrm{f}}=\frac{p_{1}-p_{2}}{\rho}+\frac{Q^{2}}{2}\left(A_{1}^{-2}-A_{2}^{-2}\right)-Z g .
$$

2.2. Geometrical Model. Due to the hard-to-measure pressure and area in real eye, numerical simulation is used to simulate the $\mathrm{AH}$ flow and assess those parameters in equation (4). The geometric models for finite element analysis, including cornea, iris, lens, and AH (Figures 3(a)-3(c)), were developed from the finite model in our previous study [18]. All the models were established with similar geometry parameters as typical ocular dimensions [25-27], with the assumption that the anterior segment was axisymmetric. Notably, different ILC distances as well as pupil diameters were used to construct these models, while the other geometric parameters remained the same. Detailed dimensions of the models are shown in Table 1. Considering the fact that obvious resistance in $\mathrm{AH}$ could be 


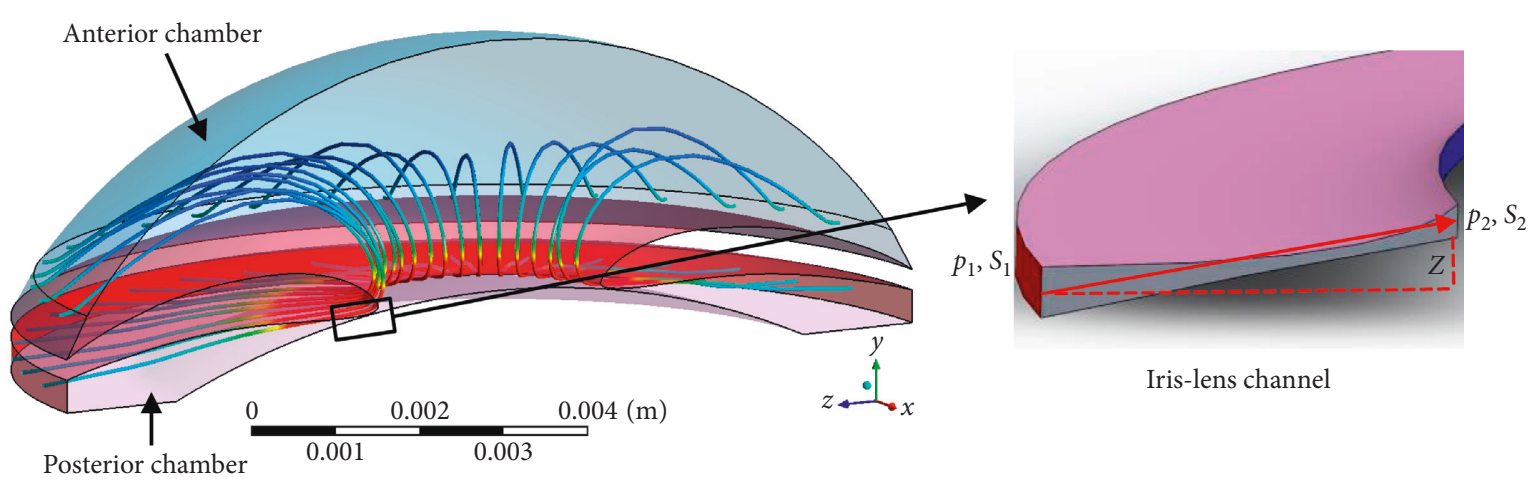

FIgure 2: The trajectories of AH flow from PC to AC and its flow through ILC (enlarged figure).

Outlet (collecting channel)

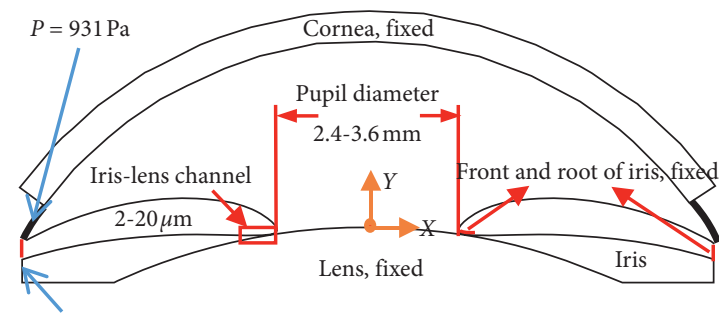

Inlet (ciliary body)

$V=3 \mu \mathrm{l} / \mathrm{min}$, normal to boundary

(a)

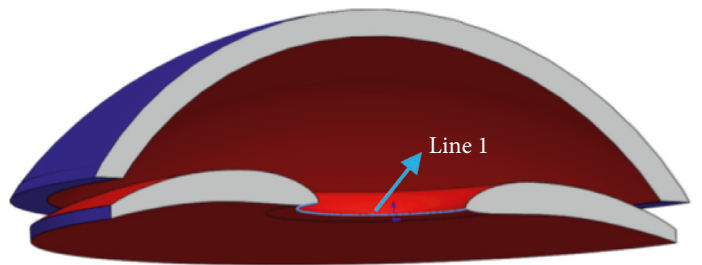

(c)

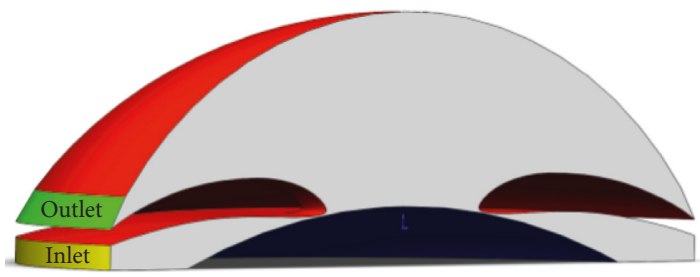

(b)

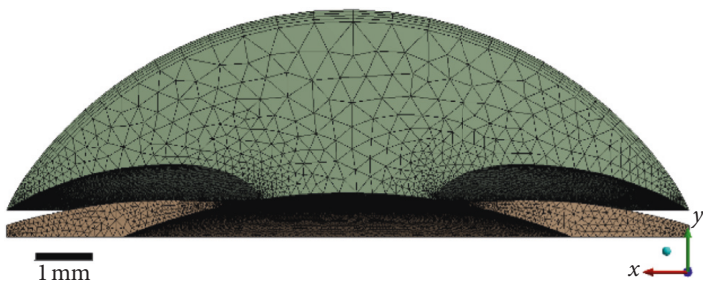

(d)

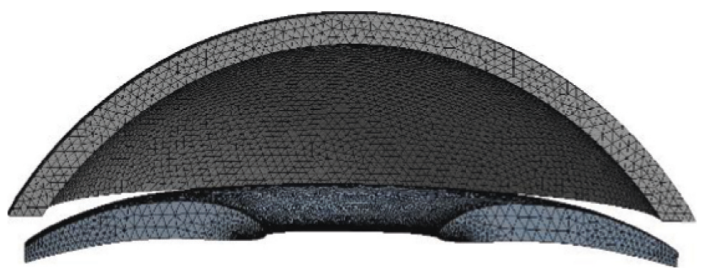

(e)

FIGURE 3: Schematic diagram of the finite element model and its computational grid: (a) 2D scheme of the model; (b) 3D scheme of AH; (c) $3 \mathrm{D}$ scheme of iris and cornea; (d) computational grids of $\mathrm{AH}$; (e) computational grids of iris and cornea.

observed when the ILC distance was under $20 \mu \mathrm{m}$ and PB was defined when the distance was less than $5 \mu \mathrm{m}[28,29]$, seven levels $(20 \mu \mathrm{m}, 15 \mu \mathrm{m}, 10 \mu \mathrm{m}, 5 \mu \mathrm{m}, 4 \mu \mathrm{m}, 3 \mu \mathrm{m}$, and $2 \mu \mathrm{m})$ of ILC distances were chosen to simulate healthy and different PB conditions. Three levels $(2.4 \mathrm{~mm}, 3 \mathrm{~mm}$, and $3.6 \mathrm{~mm})$ of pupil diameters [30] were set to simulate different pupil sizes in PACG patients. Based on full factorial design, twenty-one models were constructed and are shown in Figures 3(a)-3(c). All of these models were conducted to generate the tetrahedron mesh using ICEM CFD and Mechanical APDL (Figures 3(d) and $3(\mathrm{e})$ ). The mesh was refined, and the independency was checked. The computational grids were exported to ANSYS Workbench (ANSYS Inc., Canonsburg, PA, USA) for fluidsolid coupling simulation analysis.

2.3. Fluid-Solid Coupling Analysis. In order to calculate the $P_{\mathrm{d}}$ between surfaces $S_{1-2}$ and investigate the effect of PB on 
TABLE 1: The geometrical parameter of finite element models.

\begin{tabular}{lcc}
\hline Quantity & Finite element model & Sources \\
\hline Diameter of the anterior chamber & $13 \mathrm{~mm}$ & {$[31]$} \\
Maximum height of the chamber & $2.63 \mathrm{~mm}$ & $6.8 \mathrm{~mm}$ \\
Maximum radius of curvature of the posterior cornea & $10 \mathrm{~mm}$ & {$[31]$} \\
Radius of curvature of the natural lens & $2-20 \mu \mathrm{m}$ & {$[31]$} \\
Height of the iris-lens channel & $2.4-3.6 \mathrm{~mm}$ & {$[31]$} \\
Diameter of pupil & $30^{\circ}$ & {$[30]$} \\
Angle between cornea and iris & {$[31]$} \\
\hline
\end{tabular}

eye shape with different pupil diameters, all the finite element models were conducted to simulate AH flow by fluidsolid coupling numerical simulation. To model the motion of AH steady flow from PC to AC, the Navier-Stokes equation representing the conservation of the momentum is written as

$$
\rho(u \cdot \nabla u)=-\nabla p+\mu \nabla^{2} u+\rho g .
$$

From left to right, the terms in equation (5) represent the change in momentum caused by convection, pressure gradients, viscous diffusion, and gravity. Considering $\mathrm{AH}$ is an incompressible Newtonian viscous fluid, the continuity equation is given as

$$
\nabla \cdot u=0
$$

For the boundary conditions, the inlet (the yellow surface in Figure 3(b)) was considered as constant flux and the flux rate was set at $3 \mu \mathrm{L} / \mathrm{min}$, which was equal to the generation rate of $\mathrm{AH}$ in real eyes [32]. The outlet (the green surface in Figure 3(b)) was set as constant pressure (scleral venous pressure) for $\mathrm{AH}$ fluid. The exterior surface of lens, iris root (the blue surfaces in Figure 3(c)), and pupil (Figure 3(c) line 1) were set as fixed positions. The interface between the cornea and $\mathrm{AH}$ and the interface between iris and $\mathrm{AH}$ (the red surfaces in Figures 3(b) and 3(c)) were chosen as fluid-solid interaction surfaces to simulate the interaction between AH fluid and solid bodies [25]. Moreover, the bottom (the blue surface in Figure 3(b)) was applied to the wall boundary condition. As for the material properties, the liquid properties in the simulation were assumed to be those of pure water. The solid properties of cornea and iris were set according to the measurement of real eyes. A nonlinear elasticity model ( $2^{\text {nd }}$ Ogden model) was used to simulate the mechanical properties of iris. Cornea was considered to be a linear elastic material and iris was a nonlinear elastic material. Detailed information about material properties is shown in Table 2 .

2.4. Soft Measurement Method of PBF. Based on the result of fluid-solid coupling analysis, the $H_{f}$ in each model was calculated by equation (4). In order to investigate the effect of pupil diameters, ILC distances, and their interaction on PBF, the response surface methodology (RSM) was selected to fit the experimental data. RSM was considered as a simple and fast empirical tool to study both the effect of each individual factor and their interactions on the response variable [38, 39]. By using SPSS Statistics software, a RSM model was constructed and was optimized with a sequential quadratic programming approach. After that, analysis of variance was used to verify the significance of the optimized model and its parameters. Finally, an empirical equation was derived to predict PBF based on the measurement of ILC distance and pupil diameter.

2.5. Analysis and Calculation. The areas of surfaces $S_{1-2}$ $\left(A_{1-2}\right)$ were calculated using SolidWorks2014 software, and the pressures $\left(P_{1-2}\right)$ in those surfaces were derived from the results of fluid-solid coupling analysis by CFD-Post in ANSYS Workbench. Then, the magnitude of PBF was calculated by equation (4) according to the above parameters. The deformation of iris (De), equivalent stress, and equivalent elastic strain of iris were also obtained by CFD-Post. Because of the irregular curved surface of the iris, the anterior chamber angle can be measured by averaging the ACA values in the results of iris deformation images of repeated manual measurements.

\section{Results and Discussion}

3.1. Effects of Iris-Lens Channel Distance and Pupil Diameter on AH Flow. With various ILC distances and pupil diameters, the velocity and pressure distributions of $\mathrm{AH}$ within eyes are shown in Figure 4, where gravity was opposite to the direction of $y$-axis, representing the supine position in clinical. It was obvious that the main flux of $\mathrm{AH}$ from PC to AC was at a low velocity. However, the fluid was accelerated rapidly and the velocity achieved peak when $\mathrm{AH}$ flowed through ILC. Besides, an obvious difference in pressure between PC and AC could be observed because of the resistance of ILC, according to a previous study [40]. The maximum velocity of $\mathrm{AH}\left(V_{\max }\right)$ and the $P_{\mathrm{d}}$ between $\mathrm{PC}$ and AC were different with various ILC distances and pupil diameters. Despite the same pupil diameter $(3.6 \mathrm{~mm})$, the $V_{\text {max }}$ and $P_{\mathrm{d}}$ were $5.39 e-4 \mathrm{~m} / \mathrm{s}$ and $1.60 \mathrm{~Pa}$ when the ILC distance was $20 \mu \mathrm{m}$ (Figure 4(a)), while they became $1.72 e-3 \mathrm{~m} / \mathrm{s}$ and $257.00 \mathrm{~Pa}$ once the distance decreased to $2 \mu \mathrm{m}$ (Figure 4(b)). When the pupil diameter decreased from $3.6 \mathrm{~mm}$ to $2.4 \mathrm{~mm}$, the $V_{\max }$ and $P_{\mathrm{d}}$ increased to $2.13 e-3 \mathrm{~m} / \mathrm{s}$ and $392.00 \mathrm{~Pa}$ further, indicating both the ILC distance and diameter had effects on the $V_{\max }$ as well as $P_{\mathrm{d}}$. The previous study has demonstrated that $V_{\max }$ and $P_{\mathrm{d}}$ could influence AC morphology [41], which was also confirmed by the AC deformation in our study (Figure 4). Iris bomb and ACA blocking could be observed obviously when the ILC became narrow (Figures 4(a) and 4(b)), according to clinical trials 
TABLE 2: Material properties of AH, cornea, and iris in finite element models.

\begin{tabular}{|c|c|c|}
\hline Material properties & Value & Sources \\
\hline AH density & $1000 \mathrm{~kg} \cdot \mathrm{m}^{-3}$ & [33] \\
\hline $\mathrm{AH}$ viscosity & $0.001 \mathrm{~kg}^{-1} \cdot \mathrm{s}^{-1}$ & {$[34]$} \\
\hline AH volumetric flux secreted by ciliary body, $V$ & $3 \mu \mathrm{L} / \mathrm{min}$ & {$[32]$} \\
\hline AH outlet pressure (scleral venous pressure), $P$ & $7 \mathrm{mmHg}$ & {$[35]$} \\
\hline Cornea density & $1143 \mathrm{~kg} \cdot \mathrm{m}^{-3}$ & {$[36]$} \\
\hline Cornea Young's modulus & $1.5 \mathrm{MPa}$ & {$[36]$} \\
\hline Iris density & 1000 & {$[37]$} \\
\hline Iris $2^{\text {nd }}$ Ogden material coefficients & $\begin{array}{c}\mu_{1}=43.05 \mathrm{kPa}, \mu_{2}=37.7 \mathrm{kPa} \\
\alpha_{1}=54.255, \alpha_{2}=48.072\end{array}$ & {$[37]$} \\
\hline
\end{tabular}

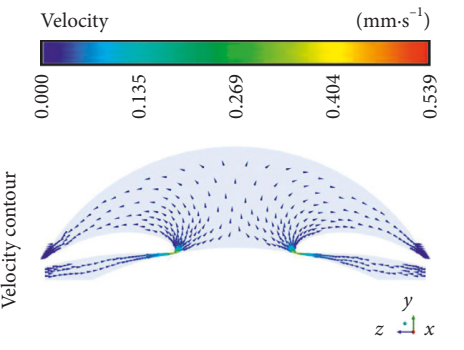

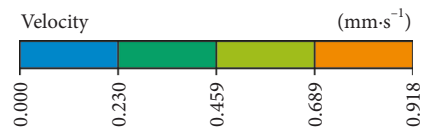

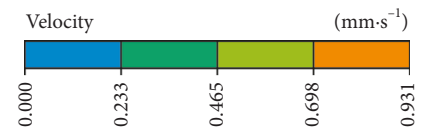
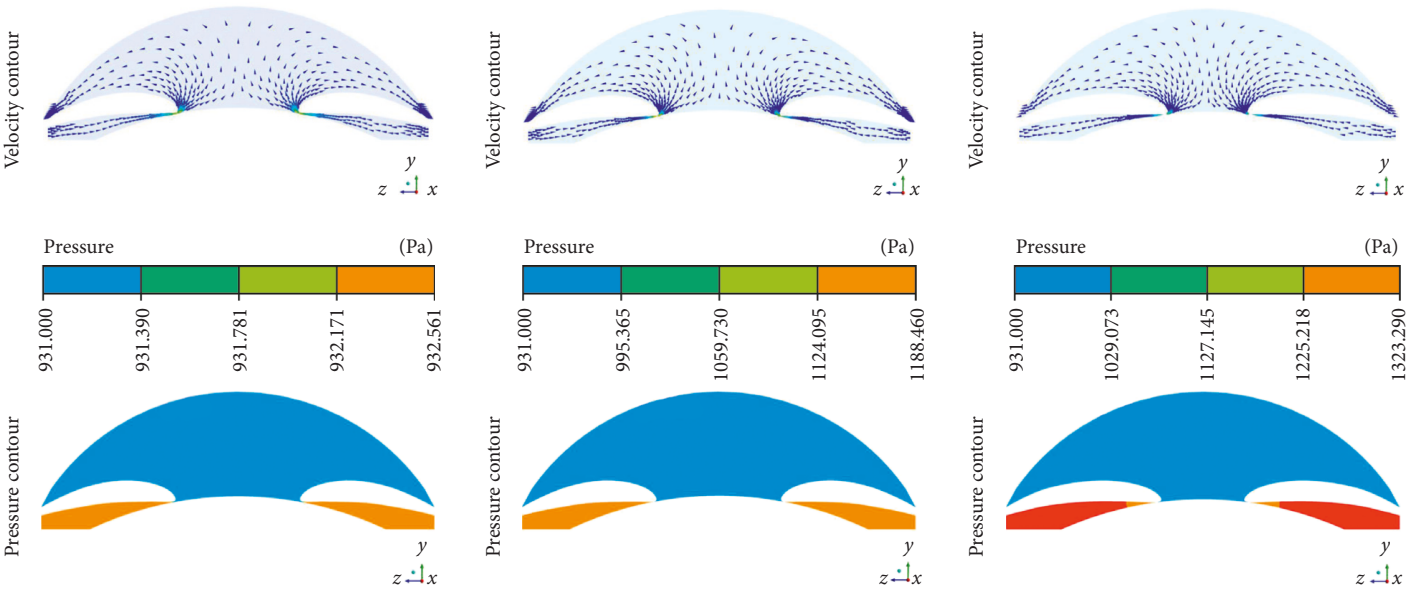

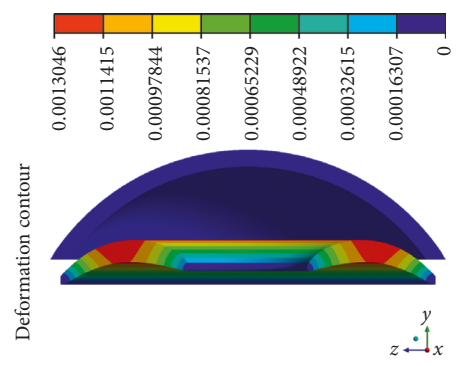

(a)
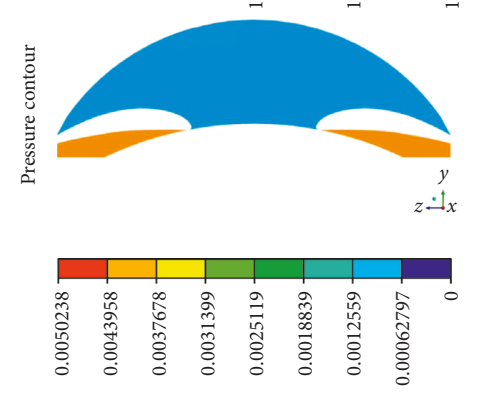

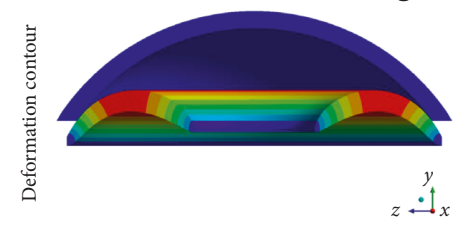

(b)
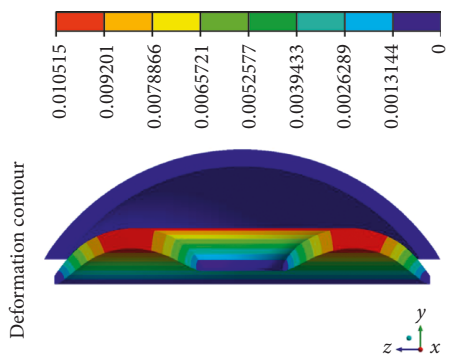

(c)

Figure 4: Contours of the velocity and pressure with various ILC distances and pupil diameters: (a) $20 \mu \mathrm{m}$ and $3.6 \mathrm{~mm}$; (b) $2 \mu \mathrm{m}$ and $3.6 \mathrm{~mm}$; (c) $2 \mu \mathrm{m}$ and $2.4 \mathrm{~mm}$.

[42]. Notably, the degree of iris bomb and ACA blocking varied with pupil diameter, indicating that pupil diameter was also an important factor to assess the risk of PB [43].

The results of $V_{\max }$ and $P_{\mathrm{d}}$ under different ILC distances and pupil diameters are shown in Figure 5. With the decrease of ILC distance, an exponential increase of $V_{\max }$ and $P_{\mathrm{d}}$ could be observed under each level of pupil diameter (Figure 5(a)). Besides, both $V_{\max }$ and $P_{\mathrm{d}}$ varied with pupil diameter, especially under narrow ILC distances (Figures 5(a) and 5(b)). Similarly, an exponential increase of ACA could be observed with the decrease of ILC distance (Figure 5(c)), indicating a great effect of $\mathrm{PB}$ on AC morphology. The effect of pupil diameter on iris deformation showed greater differences under different levels of ILC distances (Figure 5(c)). When the pupil diameter decreased from $3.6 \mathrm{~mm}$ to $2.4 \mathrm{~mm}$, ACA increased $85.48 \%$ under the ILC distance of $2 \mu \mathrm{m}$ while only $23.88 \%$ under the ILC distance of $20 \mu \mathrm{m}$. Furthermore, the curves of the relationships between $V_{\max }$ and ILC distance were not parallel under each level of pupil diameter. Similar phenomena could be observed in Figures 5(b) and 5(c), suggesting a potential interaction between ILC and pupil diameter on $V_{\max }, P_{\mathrm{d}}$, and iris deformation [44].

3.2. Soft Measurement Method of PBF. Considering the obvious effects of ILC as well as pupil diameter on the $P_{\mathrm{d}}$ and 

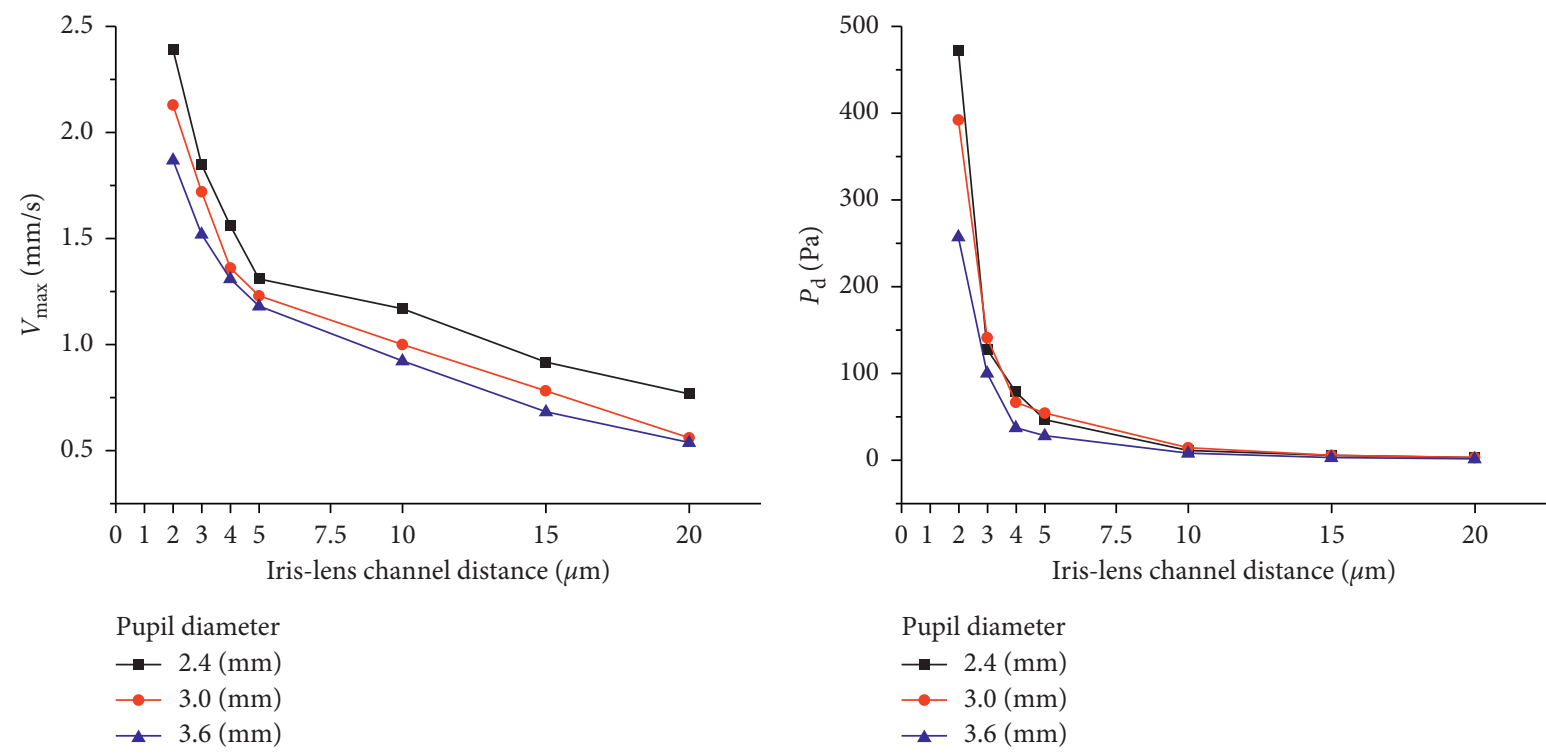

(a)

(b)

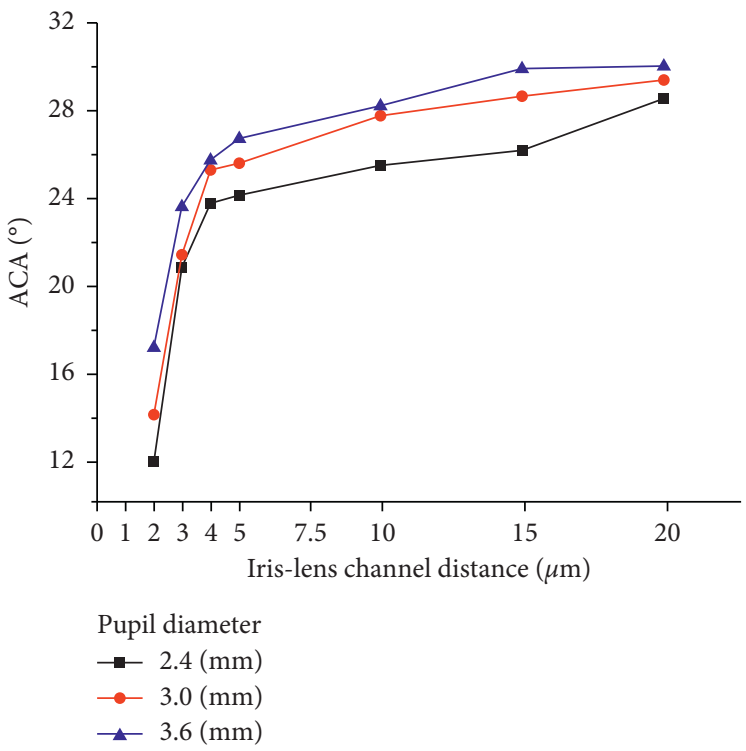

(c)

FIgURE 5: The relationship between $V_{\max }$ and ILC distance under various pupil diameter levels (a), $P_{\mathrm{d}}$ and ILC distance under various pupil diameter levels (b), and ACA and ILC distance under various pupil diameter levels (c).

AH flow velocity, it could be induced that these two factors also had an impact on PBF. The results of PBF in the twentyone models are shown in Figure 6(a). A distinct difference in $\mathrm{PBF}$ values could be observed under various ILC distances. It was only $1049.2 \mathrm{~J} / \mathrm{kg}-2342.4 \mathrm{~J} / \mathrm{kg}$ under a wide ILC $(20 \mu \mathrm{m})$ while it achieved $256449.2 \mathrm{~J} / \mathrm{kg}-472235.6 \mathrm{~J} / \mathrm{kg}$ under an extreme narrow ILC $(2 \mu \mathrm{m})$. This phenomenon verified the rationality of ILC as an indicator to assess PB risks. Moreover, similar trends could be observed in the relationship between the ILC distance and PBF under each level of pupil diameter, which appeared to be exponential growth curves. The PBF exploded when the ILC distance decreased to less than $5 \mu \mathrm{m}$, verifying the reliability of $5 \mu \mathrm{m}$ as the threshold of PB in clinical [28, 29]. Furthermore, the
PBF values increased in different degrees under various ILC distances with the same change of pupil diameter from $3.6 \mathrm{~mm}$ to $2.4 \mathrm{~mm}$, showing a potential interaction relationship between ILC distance and pupil diameter on PBF.

Despite high accuracy to assess PBF using finite element analysis, it was not suitable for clinical application because of complex operation and high cost. Therefore, it was necessary to establish a soft measurement method to evaluate PBF. Considering the exponential distributions of PBF under each pupil diameter level, the logarithm of PBF value was used to develop a regression model. Besides, ILC ratio and diameter ratio, defined by the ratio of the measured ILC distance and pupil diameter to normal values (ILC distance: $20 \mu \mathrm{m}$; pupil diameter: $3 \mathrm{~mm}$ ), were calculated to eliminate the 


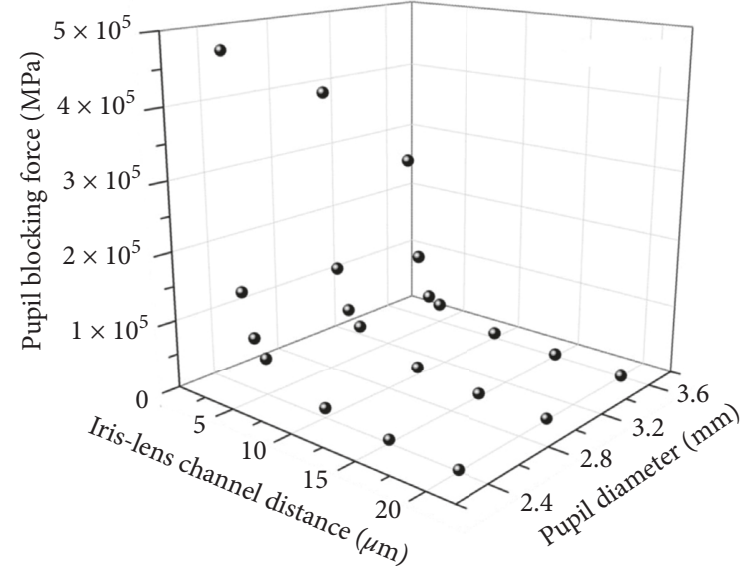

- $\operatorname{PBF}(\mathrm{MPa})$

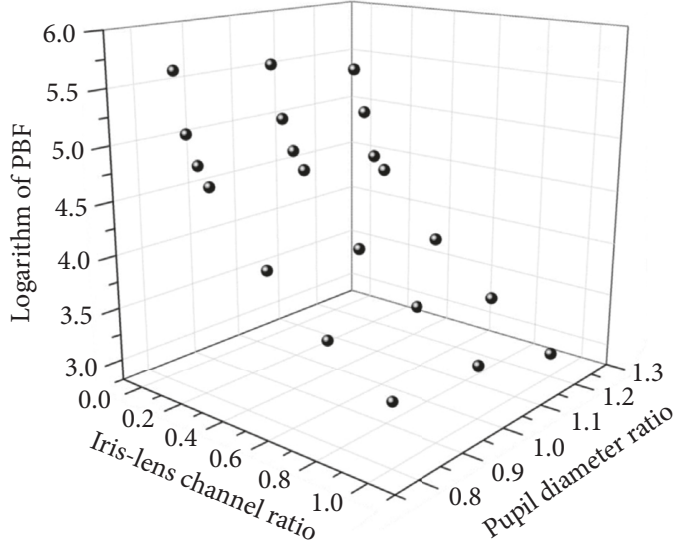

- LgPBF

(a)

(b)

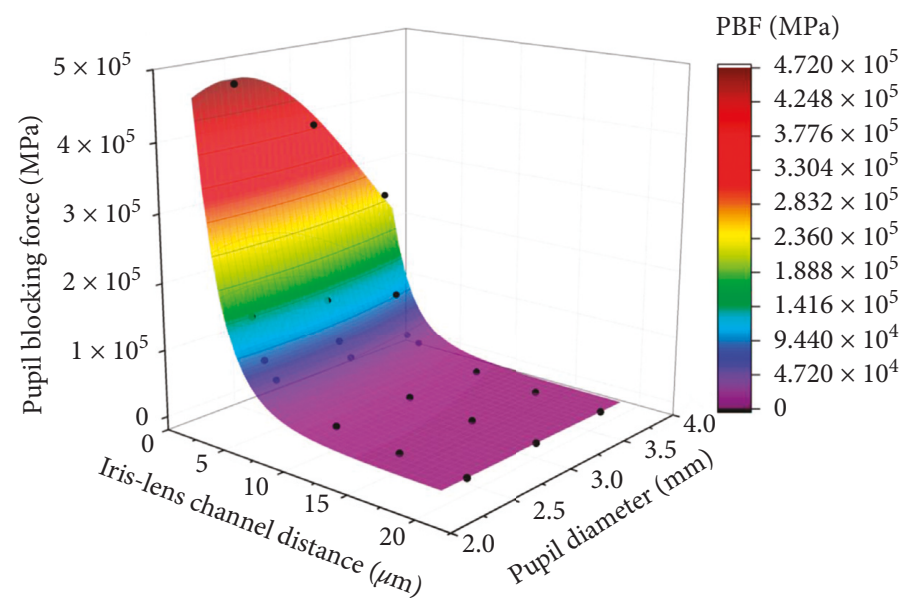

(c)

FIGURE 6: The experimental data and predicted model of PBF with various ILC distances and pupil diameters: (a) the relationship between PBF and ILC distance as well as pupil diameter; (b) the relationship between logarithmic PBF and ILC ratio as well as diameter ratio; (c) the $3 \mathrm{D}$ response surface plot and experimental data.

discrepancy in magnitude. The relationships between logarithmic PBF and the ILC ratio as well as the diameter ratio are shown in Figure 6(b). By applying multiple regression analysis on these data, a second-order polynomial equation for the logarithmic PBF was obtained as follows:

$$
\begin{aligned}
y= & 2.328 x_{1}^{2}-1.749 x_{2}^{2}-0.263 x_{1} x_{2}-4.573 x_{1} \\
& +2.910 x_{2}+4.682
\end{aligned}
$$

where $y$ represents the logarithmic PBF and $x_{1}$ and $x_{2}$ are the ILC ratio and pupil diameter ratio, respectively. The result of variance analysis showed that the determination coefficient of this model achieved 0.973 , implying approximately $98 \%$ of the variability in logarithmic PBF could be expressed by the model. Therefore, the empirical relationship between $\mathrm{PBF}$ value and the measured parameters (including ILC distance and pupil diameter) was quantified using the following equation:

$$
Y=48083.935 \cdot 10^{\left(0.00596 X_{1}-0.194 X_{2}-0.00438 X_{1} X_{2}-0.229 X_{1}+0.970 X_{2}\right)} \text {, }
$$

where $Y$ represents the value of $\mathrm{PBF}(\mathrm{J} / \mathrm{kg})$ and $X_{1}$ and $X_{2}$ are, respectively, the measured ILC distance $(\mu \mathrm{m})$ and pupil diameter $(\mathrm{mm})$.

3.3. Effect of PBF on AC Morphology. In order to testify the feasibility of $\mathrm{PBF}$ as an indicator to assess the risks of $\mathrm{PB}$ in clinical, the relationship between $\mathrm{PBF}$ and variation of ACA was investigated and is shown in Figure 7. ACA increased with $\mathrm{PBF}$, and a linear relationship was observed between ACA and PBF. The determination coefficient achieved 0.92 , indicating that more than $92 \%$ variation in ACA could be explained by PBF. Similar phenomenon could be observed in the relationship between deformation of iris and PBF. With the increase of PBF, the deformation of iris increased linearly. The determination coefficient of the fitted line was 0.95, suggesting the linear model was able to express more than $95 \%$ of the variability in iris deformation. Iris deformation and variation in ACA were considered as the pathogenic mechanism of PACG [45]. Increased iris deformation could decrease AC depth and narrow ACA, 


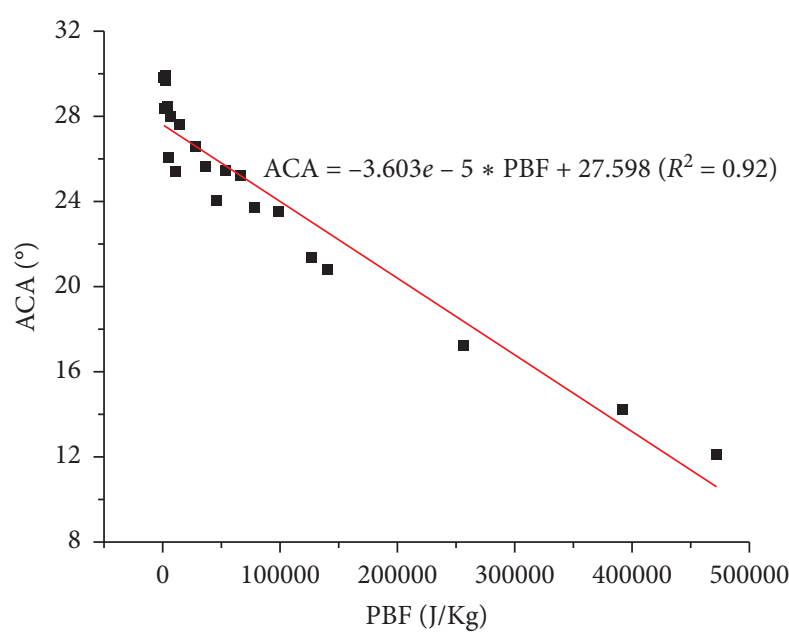

- Experimental data

— Fitting line

(a)

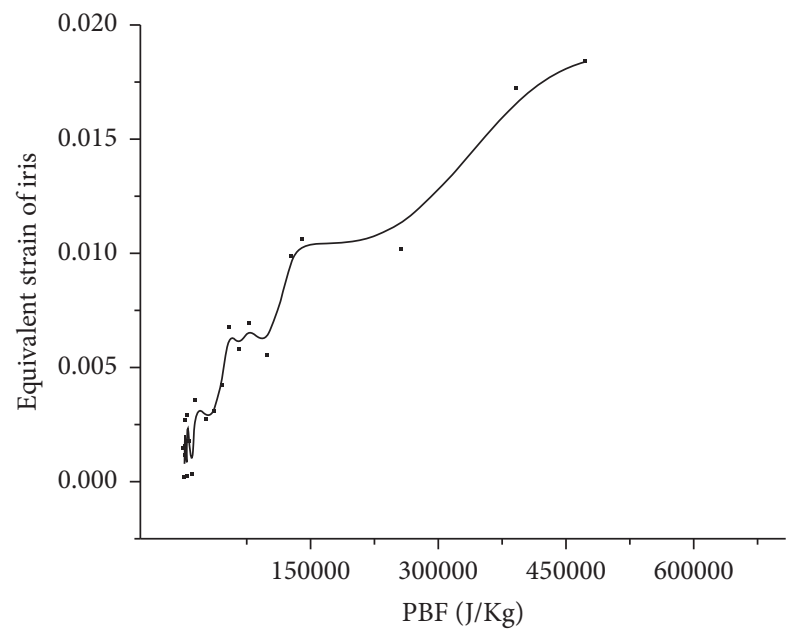

(c)

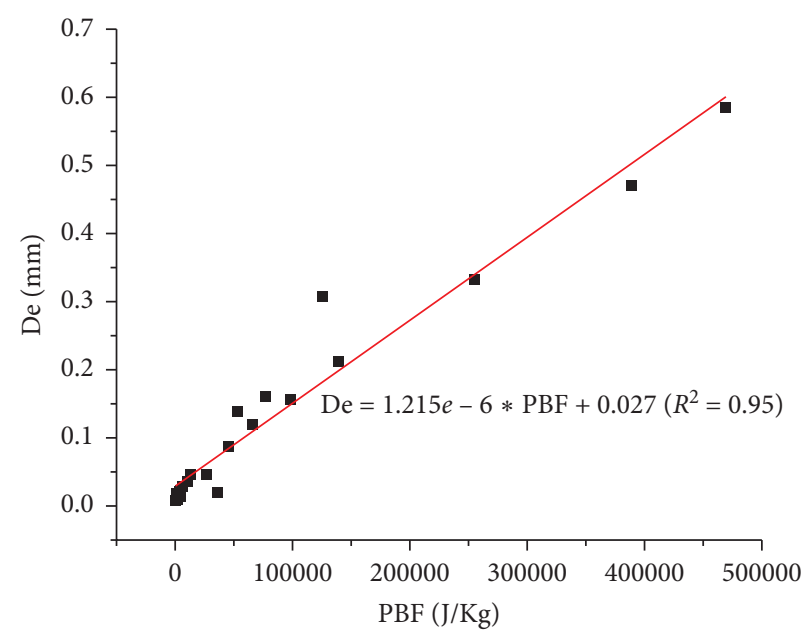

- Experimental data

- Fitting line

(b)

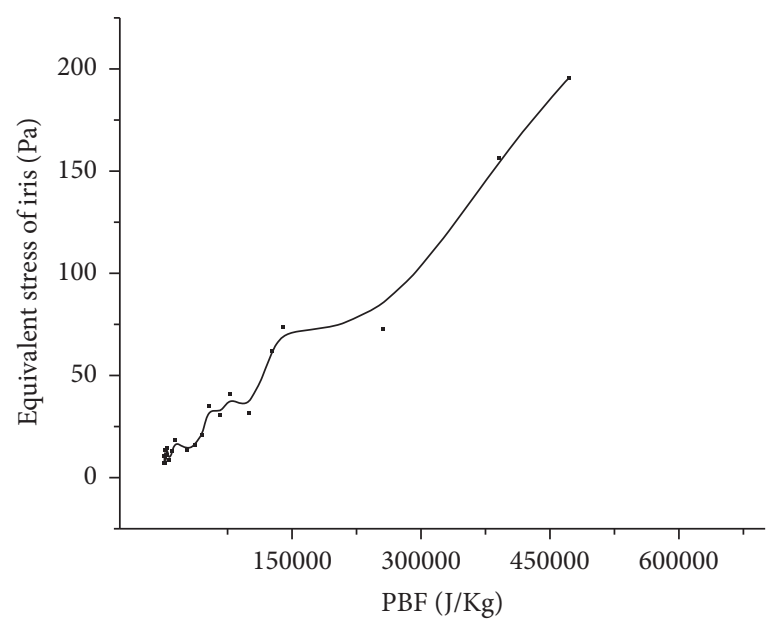

(d)

FIGURE 7: The relationships and fitting lines between ACA and PBF (a) and De and PBF (b); the relationships between equivalent elastic strain and PBF (c) and equivalent elastic stress and PBF (d).

resulting in a severe obstruction of AH outflow, which could cause high IOP in eye and induce acute attack of angleclosure glaucoma. Therefore, PBF could be utilized as an indicator to assess the effect of $\mathrm{PB}$ on $\mathrm{AC}$ morphology and evaluate its risks. Furthermore, obvious tendency of increase in equivalent elastic strain and equivalent stress of iris could be observed with the increase of PBF. The equivalent elastic strain of iris represented its deformability, which could be utilized to evaluate the risk of iris bomb. Increased equivalent stress of iris under high PBF could hasten the detachment of epithelial cells in iris, which might cause an obstruction in $\mathrm{AC}$ and rise the resistance of $\mathrm{AH}$ outflow [46], verifying the reliability of $\mathrm{PBF}$ to assess the risks of $\mathrm{BP}$.

According to clinical experience, obvious deformation of iris and narrowing of ACA could be observed when ILC distance decreased to $5 \mu \mathrm{m}$ with a $3 \mathrm{~mm}$ pupil diameter. The value of PBF under that pathology condition was $45835.6 \mathrm{~J} /$ $\mathrm{kg}$. Considering the effect of pupil diameter on PBF, the value should fluctuate in the range from $27849.2 \mathrm{~J} / \mathrm{kg}$ to $53843.4 \mathrm{~J} / \mathrm{kg}$. As mentioned in Section 3.2, a quadratic polynomial equation has been solved to explain the variation in PBF, and a second-order empirical model has been developed to provide a good estimate of PBF. Based on the linear phenomena between $\mathrm{PBF}$ and $\mathrm{ACA}$ as well as $\mathrm{PBF}$ and $\mathrm{De}$, it was reasonable to use the $\mathrm{PBF}$ value of $27000 \mathrm{~J} / \mathrm{kg}$ as $\mathrm{PB}$ risk threshold in clinical. Further study will focus on the optimization of the empirical model of PBF to enlarge the range of pupil diameter.

\section{Conclusions}

This study presented a simple and practical method to assess PBF by the measurement of ILC distance and pupil diameter, which were accessible parameters in clinical. The results demonstrated that the ILC distance had an obvious effect on PBF. In a specific range of pupil diameter from 
$2.4 \mathrm{~mm}$ to $3.6 \mathrm{~mm}$, the ACA became narrow with the decrease of the diameter. The quantitative analysis of PBF in this study could be used to research PACG pathological correlation and its pathogenesis, so as to provide a threshold for clinical diagnosis of PACG.

\section{Data Availability}

The data used to support the findings of this study are available from the corresponding author upon request.

\section{Conflicts of Interest}

The authors declare that there are no conflicts of interest regarding the publication of this paper.

\section{Acknowledgments}

This work was supported by the National Natural Science Foundation of China (nos. 10802053 and 31570952) and Beijing Natural Science Foundation (no. 7152022).

\section{References}

[1] D. F. Sena and K. Lindsley, "Neuroprotection for treatment of glaucoma in adults," Cochrane Database of Systematic Reviews, vol. 1, article CD006539, 2013.

[2] R. N. Weinreb, T. Aung, and F. A. Medeiros, "The pathophysiology and treatment of glaucoma: a review," JAMA, vol. 311, no. 18, pp. 1901-1911, 2014.

[3] D. Pascolini and S. P. Mariotti, "Global estimates of visual impairment: 2010," British Journal of Ophthalmology, vol. 96, no. 5, pp. 614-618, 2012.

[4] P. Song, J. Wang, K. Bucan, E. Theodoratou, I. Rudan, and K. Y. Chan, "National and subnational prevalence and burden of glaucoma in China: a systematic analysis," Journal of Global Health, vol. 7, no. 2, 2017.

[5] J. van der Schoot, The Complexity of Diagnosing and Monitoring Glaucoma, Erasmus University Rotterdam, Rotterdam, Netherlands, 2017.

[6] E. W. Chan, X. Li, Y.-C. Tham et al., "Glaucoma in Asia: regional prevalence variations and future projections," British Journal of Ophthalmology, vol. 100, no. 1, pp. 78-85, 2016.

[7] C. Y. Cheung, S. L. Li, N. Chan et al., "Factors associated with long-term intraocular pressure fluctuation in primary angle closure disease: the CUHK PACG longitudinal (CUPAL) study," Journal of Glaucoma, vol. 27, p. 1, 2018.

[8] B. S. Shastry, "Genetic susceptibility to primary angle closure glaucoma (PACG)," Discovery Medicine, vol. 15, no. 80, pp. 17-22, 2013.

[9] E. C. Huang and V. H. Barocas, "Active iris mechanics and pupillary block: steady-state analysis and comparison with anatomical risk factors," Annals of Biomedical Engineering, vol. 32, no. 9, pp. 1276-1285, 2004.

[10] C. R. Ethier, M. Johnson, and J. Ruberti, "Ocular biomechanics and biotransport," Annual Review of Biomedical Engineering, vol. 6, no. 1, pp. 249-273, 2004.

[11] J. Caprioli, "The ciliary epithelia and aqueous humor," in Adlers Physiology of the Eye, pp. 228-247, Mosby, St. Louis, MO, USA, 1992.

[12] S. T. Fontana and R. F. Brubaker, "Volume and depth of the anterior chamber in the normal aging human eye," Archives of Ophthalmology, vol. 98, no. 10, pp. 1803-1808, 1980.
[13] F. J. Macri, S. J. Cevario, and J. Halel, "Timolol inhibition of isoproterenol action-1. Effects on aqueous humor production and IOP," General Pharmacology: The Vascular System, vol. 11, no. 2, pp. 207-211, 1980.

[14] H. A. Quigley, D. S. Friedman, and N. G. Congdon, "Possible mechanisms of primary angle-closure and malignant glaucoma," Journal of Glaucoma, vol. 12, no. 2, pp. 167-180, 2003.

[15] C. R. Canning, M. J. Greaney, J. N. Dewynne, and A. D. Fitt, "Fluid flow in the anterior chamber of a human eye," Mathematical Medicine and Biology, vol. 19, no. 1, pp. 31-60, 2002.

[16] R. Mapstone, "Forces determining pupil size," Experimental Eye Research, vol. 10, no. 1, pp. 47-52, 1970.

[17] T. Kondo and M. Miura, "A method of measuring pupilblocking force in the human eye," Graefe's Archive for Clinical and Experimental Ophthalmology, vol. 225, no. 5, pp. 361-364, 1987.

[18] W. Wang, X. Qian, H. Song, M. Zhang, and Z. Liu, "Fluid and structure coupling analysis of the interaction between aqueous humor and iris," Biomedical Engineering Online, vol. 15, no. S2, p. 133, 2016.

[19] W. Wang, X. Qian, Q. Li et al., "Experimental study of aqueous humor flow in a transparent anterior segment phantom by using PIV technique," Molecular \& Cellular Biomechanics, vol. 16, no. 1, pp. 59-74, 2019.

[20] R. Mapstone, "Acute shallowing of the anterior chamber," British Journal of Ophthalmology, vol. 65, no. 7, pp. 446-451, 1981.

[21] J. S. Tiedeman, "A physical analysis of the factors that determine the contour of the Iris," American Journal of Ophthalmology, vol. 111, no. 3, pp. 338-343, 1991.

[22] P. Tarongoy, C. L. Ho, and D. S. Walton, "Angle-closure glaucoma: the role of the lens in the pathogenesis, prevention, and treatment," Survey of Ophthalmology, vol. 54, no. 2, pp. 211-225, 2009.

[23] R. Millar, "Flow resistance," in Encyclopedia of Earth Science, pp. 476-478, Springer, Berlin, Heidelberg, Germany, 1978.

[24] C. J. Pavlin, K. Harasiewicz, and F. S. Foster, "Ultrasound biomicroscopy of anterior segment structures in normal and glaucomatous eyes," American Journal of Ophthalmology, vol. 113 , no. 4, pp. 381-389, 1992.

[25] R. Amini and V. H. Barocas, "Reverse pupillary block slows iris contour recovery from corneoscleral indentation," Journal of Biomechanical Engineering, vol. 132, article 071010, 2010.

[26] R. Amini and V. H. Barocas, "Anterior chamber angle opening during corneoscleral indentation: the mechanism of whole eye globe deformation and the importance of the Limbus (vol. 50, pg. 5288, 2009)," Investigative Ophthalmology \& Visual Science, vol. 54, p. 7323, 2013.

[27] R. Amini, S. Jouzdani, and V. H. Barocas, "Increased iris-lens contact following spontaneous blinking: mathematical modeling," Journal of Biomechanics, vol. 45, no. 13, pp. 2293-2296, 2012.

[28] R. M. Caronia, J. M. Liebmann, Z. Stegman, J. Sokol, and R. Ritch, "Increase in iris-lens contact after laser iridotomy for pupillary block angle closure," American Journal of Ophthalmology, vol. 22, no. 1, pp. 53-57, 1996.

[29] H. A. Quigley, D. M. Silver, D. S. Friedman et al., "Iris crosssectional area decreases with pupil dilation and its dynamic behavior is a risk factor in angle closure," Journal of Glaucoma, vol. 18, no. 3, pp. 173-179, 2009.

[30] M. Heather, X. Guihua, and L. Christopher Kai-Shun, "Imaging the iris with swept-source optical coherence tomography: relationship between iris volume and primary angle closure," Ophthalmology, vol. 120, no. 12, pp. 2517-2524, 2013. 
[31] R. Repetto, J. O. Pralits, J. H. Siggers, and P. Soleri, "Phakic iris-fixated intraocular lens placement in the anterior chamber: effects on aqueous flow," Investigative Opthalmology \& Visual Science, vol. 56, no. 5, pp. 3061-3068, 2015.

[32] V. H. Barocas and E. C. Huang, "Aqueous humor and iris mechanics in pupillary block and primary angle-closure glaucoma," Investigative Ophthalmology \& Visual Science, vol. 45, no. 13, p. 5033, 2004.

[33] A. Villamarin, S. Roy, R. Hasballa, O. Vardoulis, P. Reymond, and N. Stergiopulos, "3D simulation of the aqueous flow in the human eye," Medical Engineering \& Physics, vol. 34, no. 10, pp. 1462-1470, 2012.

[34] S. Kumar, S. Acharya, R. Beuerman, and A. Palkama, "Numerical solution of ocular fluid dynamics in a rabbit eye: parametric effects," Annals of Biomedical Engineering, vol. 34, no. 3, pp. 530-544, 2006.

[35] C. A. Strohmaier, H. A. Reitsamer, and J. W. Kiel, "Episcleral venous pressure and IOP responses to central electrical stimulation in the rat," Investigative Opthalmology \& Visual Science, vol. 54, no. 10, pp. 6860-6866, 2013.

[36] J. D. Stitzel, S. M. Duma, J. M. Cormier, and I. P. Herring, "A nonlinear finite element model of the eye with experimental validation for the prediction of globe rupture," Stapp Car Crash Journal, vol. 46, pp. 81-102, 2002.

[37] K. Zhang, X. Qian, X. Mei, and Z. Liu, "An inverse method to determine the mechanical properties of the iris in vivo," Biomedical Engineering Online, vol. 13, no. 1, p. 66, 2014.

[38] G. E. P. Box and N. R. Draper, Empirical Model-Building and Response Surfaces, Wiley, New York, NY, USA, 1987.

[39] S. J. Kalil, F. Maugeri, and M. I. Rodrigues, "Response surface analysis and simulation as a tool for bioprocess design and optimization," Process Biochemistry, vol. 35, no. 6, pp. 539-550, 2000.

[40] M. Dvoriashyna, R. Repetto, M. R. Romano, and J. H. Tweedy, "Aqueous humour flow in the posterior chamber of the eye and its modifications due to pupillary block and iridotomy," Mathematical Medicine \& Biology A Journal of the IMA, vol. 35, no. 4, pp. 447-467, 2017.

[41] J. H. Siggers and C. Ross Ethier, "Fluid mechanics of the eye," Annual Review of Fluid Mechanics, vol. 44, no. 1, pp. 347-372, 2012.

[42] R. N. Weinreb and P. T. Khaw, "Primary open-angle glaucoma," The Lancet, vol. 363, no. 9422, pp. 1711-1720, 2004.

[43] A. Martucci, M. Cesareo, D. Napoli et al., "Evaluation of pupillary response to light in patients with glaucoma: a study using computerized pupillometry," International Ophthalmology, vol. 34, no. 6, pp. 1241-1247, 2014.

[44] J. Stamey, "Modern mathematical Statistics with applications," The American Statistician, vol. 62, no. 4, p. 358, 2008.

[45] N. Zebardast, S. Kavitha, P. Krishnamurthy et al., "Changes in anterior segment morphology and predictors of angle widening after laser iridotomy in south Indian eyes," Ophthalmology, vol. 123, no. 12, pp. 2519-2526, 2016.

[46] K. Yuichi, O. Tetsuro, U. Tomohiko, and S. Jun, "Effect of shear stress on attachment of corneal endothelial cells in association with corneal endothelial cell loss after laser iridotomy," Cornea, vol. 24, no. 1, pp. S55-S58, 2005. 


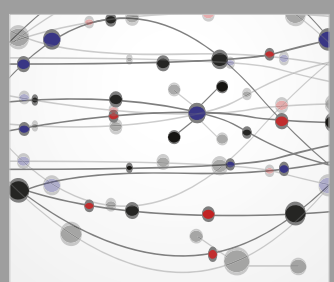

The Scientific World Journal
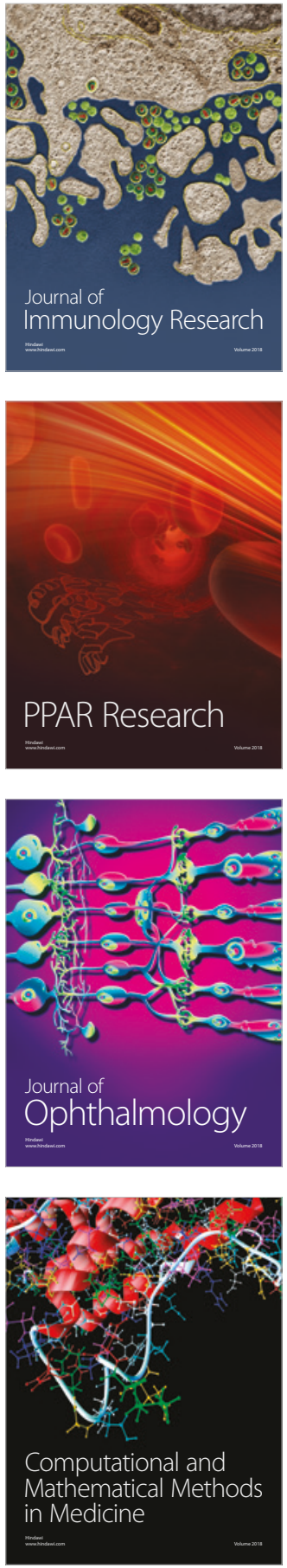

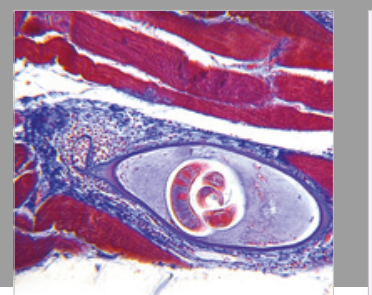

Gastroenterology Research and Practice

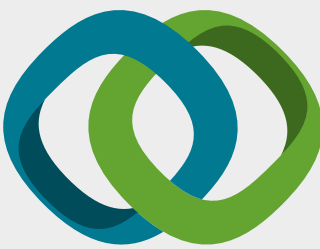

\section{Hindawi}

Submit your manuscripts at

www.hindawi.com
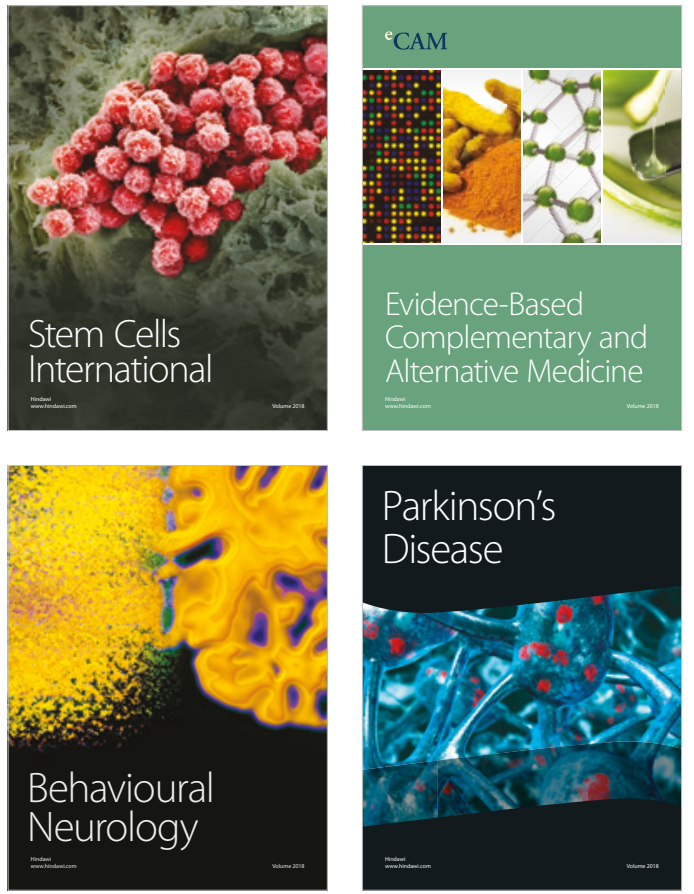

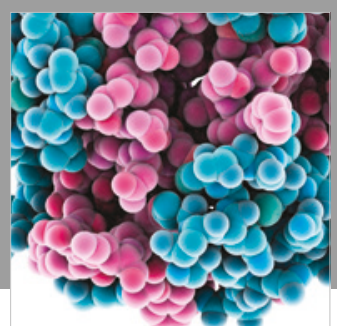

ournal of

Diabetes Research

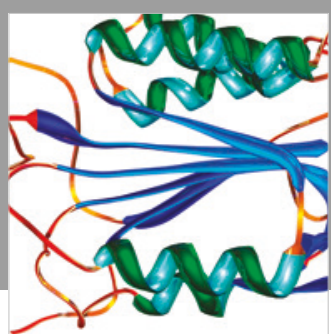

Disease Markers
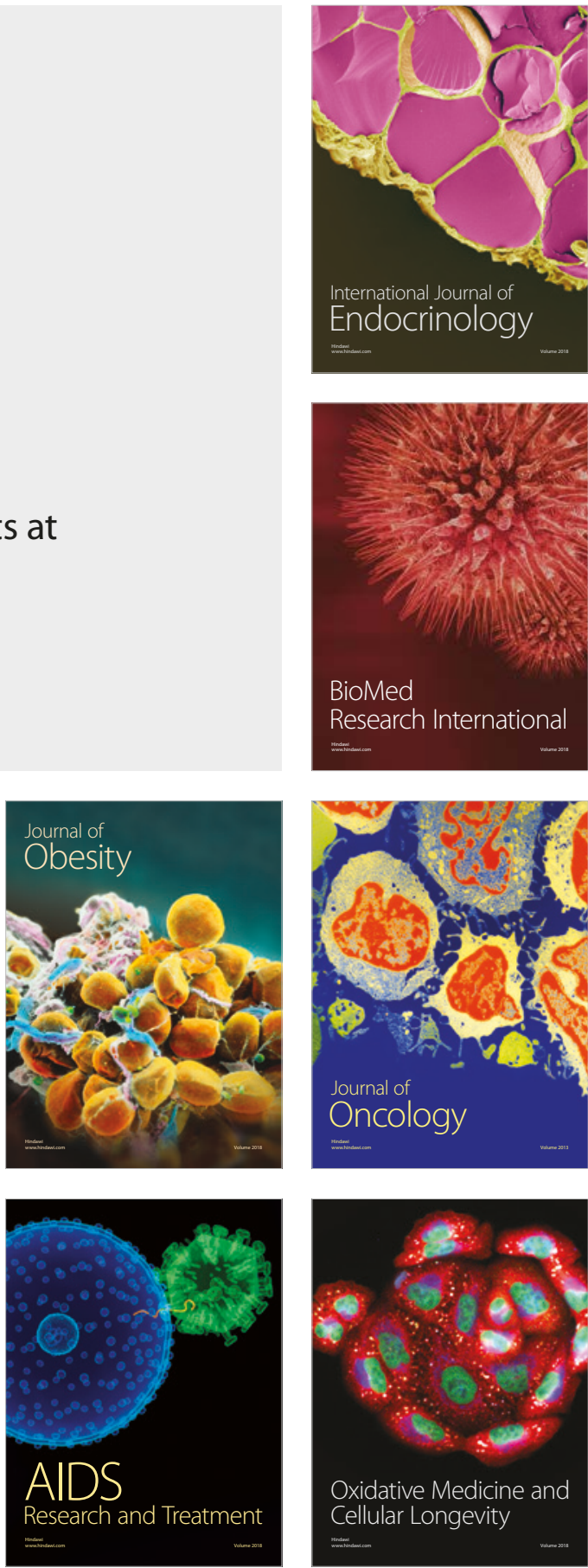